\title{
2018 Special Reviewers Thank You
}

Published online: 7 January 2019

(C) The Society for In Vitro Biology 2019

In addition to the Associate and Reviewing Editors, we thank the following for contributing to the review of manuscripts for 2018:

Ruth Appeltant

David W. Barnes

Nadhim Bayatti

Chandra Boosani

David R. Canning

Carol D. Chitko-Mckown

Matheus O. Costa

Gertrude-Emilia Costin

Zhongshan Chen

Stephanie DeWitte-Orr

Shin Enozawa

Ann M. Fallon

Michael J. Fay

Tsuyoshi Fujita

Cynthia L. Goodman

James Grasela

Alma L. Guerrero-Barrera

David W. Hammers

Zhi-Ming Han

John W. Harbell

Robert L. Harrison

Yohei Hayashi

Yasutaka Hayashido

Yukihito Higashi

Paul Higgins

Keisuke Hitachi

Masahiko Honda

Hiroyoshi Hoshi

Heng-Wei Hsu

$\mathrm{Nan} \mathrm{Hu}$

Shu-Chen Hung

Wayne Hunter

Sittipon Intarapat

Naozumi Ishimaru

Akira Ishizaki

Md Robin Nabiul Islam
Fumiko Itoh

Kenji Izumi

Yoshihiko Kakinuma

Ryu-taro Kamijo

Takashi Kanematsu

Masamoto Kanno

Koichi Kato

Hitoshi Kawamata

Takeshi Kawamoto

Kazuhiro Kawamura

Suresh Dinkar Kharche

AeRang Kim

Yasuhiro Kosuge

Yasusei Kudo

Takahiro Kunisada

Koh-ichi Kuremoto

Yang Lin

Yongshun Lin

Shinji Matsumoto

Adele Miller

Yosuke Nagata

Yukio Nakamura

Mahito Nakanishi

Koichi Nakashiro

Hideki Nakayama

Sonia Néron

Ken-ichi Nishijima

Akio Nishikawa

Takeshi Omasa

Kiyoshi Ohnuma

Jun Peng

Phuc H. Pham

Austin Prater

Paul Price

Syeda Kiran Riaz

Joseph A. Ringbauer
Shivendu Ranjan

Vincent Raquin

D. Sahin

Masakiyo Sakaguchi

Tetsushi Sakuma

Eri Sasabe

Tomohiro Sawa

Hideki Shiba

Tsuyoshi Shimo

Tomoaki Shintani

Mahipal Singh

Hideo Shigeishi

Ai Shima

Bill Smith

Silvia Stacchiotti

Mika Suga

Yutaka Sugihara

Satoru Suzuki

Shigeki Suzuki

Brad L. Upham

Katsuhiro Uzawa

Fen Wang

John Wise

Qilin Xu

Naoki Yamamoto

Akihiro Yamashita

Xiao Yang

Shen Yin

Yuji Yoshiko

Katsuhiko Yoshimoto

Hongwei Zhang

Jue Zhang

Qunzhou Zhang

Yan Zhang

Ying Zhang 